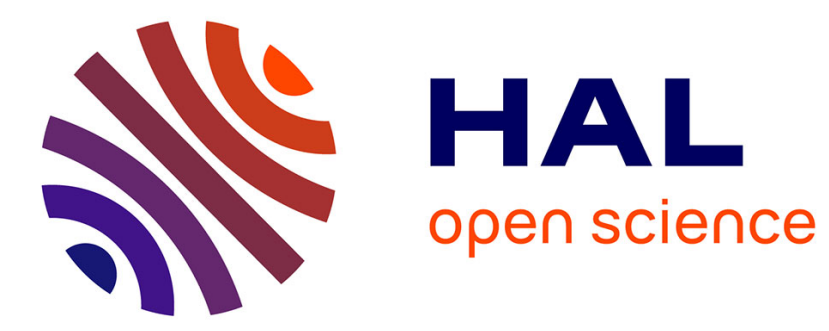

\title{
Time sub-optimal nonlinear PI and PID controllers applied to longitudinal headway car control \\ Minh-Duc Hua, Claude Samson
}

\section{To cite this version:}

Minh-Duc Hua, Claude Samson. Time sub-optimal nonlinear PI and PID controllers applied to longitudinal headway car control. [Research Report] 2010, pp.17. inria-00545646

\section{HAL Id: inria-00545646 \\ https://hal.inria.fr/inria-00545646}

Submitted on 7 Feb 2011

HAL is a multi-disciplinary open access archive for the deposit and dissemination of scientific research documents, whether they are published or not. The documents may come from teaching and research institutions in France or abroad, or from public or private research centers.
L'archive ouverte pluridisciplinaire HAL, est destinée au dépôt et à la diffusion de documents scientifiques de niveau recherche, publiés ou non, émanant des établissements d'enseignement et de recherche français ou étrangers, des laboratoires publics ou privés. 


\title{
Time sub-optimal nonlinear PI and PID controllers applied to longitudinal headway car control
}

\author{
Minh-Duc Hua, Claude Samson
}

\begin{abstract}
Simple nonlinear PI and PID controllers combining time-(sub)optimality with linear control robustness and anti wind-up properties are presented. For illustration purposes, the proposed PID solution is applied to the longitudinal headway control of a vehicle following another vehicle.
\end{abstract}

\section{Keywords}

Time (sub)-optimal control; Nonlinear PI, PID; Anti wind-up; Conditional integrator; Nested saturation; Longitudinal headway car control.

\section{INTRODUCTION}

Proportional-integral (PI) and proportional-integral-derivative (PID) controllers are at the heart of control engineering practice and, owing to their relative simplicity and satisfactory performance for a wide range of processes, have become the standard controllers used by industry. Following an estimation of [5], perhaps only $5-10 \%$ of man-implemented control loops cannot be controlled by single-input single-output (SISO) PI or PID controllers. However, this widespread usage also goes with numerous problems due to either poor tuning practice or limited capabilities offered by standard PI-PID schemes. These problems have in turn periodically revived the interest from the academic research community in order to work out complementary explanations and solutions [1], [10]. In particular, a well-known source of degradation of performance is the occurrence of control saturation, when the boundedness of the "physical" control that can be applied to the system under consideration is no longer compatible with the application of the (theoretically unbounded) calculated control value. This has the consequence of invalidating the performance index established on the assumption of linearity of the controlled system, and can give rise to various undesired (and unnecessary) effects such as multiple bouncing between minimal and maximal values of the control, and important overshoots of the regulated error variables. The so-called integrator wind-up phenomenon, which worsens the overshoot problem and the reduction of which still motivates various research studies [1], [12], is also commonly presented as a consequence of control saturation combined with the integral action incorporated in the control law in order to compensate for unknown (slowly varying) additive perturbations.

Compared to the already huge corpus of studies devoted to PI and PID controllers, the present paper has the limited ambition of proposing new nonlinear versions of these controllers that attempt to combine the constraints of control saturation with i) the objective of optimizing the control action in order to reduce the size of initially large tracking errors as fast as possible, and ii) the design of integral action terms with limited wind-up effects. The former issue is close to the line of research on "proximate" timeoptimal for linear systems admitting closed-form time-optimal solutions [15], [9], [11]. The present study is restricted to the simplest first and second order linear systems. 
In particular, continuous nonlinear proportional $(\mathrm{P})$ and proportional-derivative $(\mathrm{PD})$ state feedbacks, which depend continuously on an extra-parameter whose convergence to infinity yields the discontinuous time-optimal controls for these systems, will be derived and will form the cores of the nonlinear PI and PID controllers proposed subsequently. As for the latter issue, it is related to the work on anti-windup and "conditional integrators" as exemplified in [6], [12]. The present work is also related to the theme of bounded control design based on the use of nested saturation functions [13], [7], [14], with the same concern of proving global asymptotic stability of the desired set-point, but with a different way of designing the control solutions. In the second part of the paper, the proposed nonlinear PID controller is applied to the longitudinal headway control of a car following a leader. The reason for choosing this application is its good fit with the design constraints and objectives imposed on the control and its performance; namely i) the existence of different bounds on the car's acceleration and deceleration capabilities, ii) control effectiveness in terms of time of convergence to the desired inter-distance between the two vehicles, iii) absence of bouncing transients -for the comfort of the passengers, fuel economy, and reduced wear-off of mechanical parts-, and iv) very small overshoot in order to avoid collisions with the leader. This type of application has also motivated numerous studies in the last two decades, see for instance [3], [16], [8] among many other contributions. The results here presented are based on a simple model of the system's dynamics which would obviously call for several refinements of practical relevance, and they by no means aim at covering the subject in depth. The purpose is just to point out a novel and simple PID solution which basically addresses the same issues as in [3], with the economy of a switching strategy, and that experts on the subject might consider in the future.

\section{ReCAlls: Time-OPtimal CONTROLS (TOC) FOR FIRST-ORDER AND SECOND-ORDER INTEGRATORS AND CONTINUOUS FEEDBACK APPROXIMATIONS}

\section{A. First-order system}

Let $M>0$ and $m<0$ denote two real numbers. In what follows $s a t_{m}^{M}$ denotes the saturation function defined on $\mathbb{R}$ by

$$
\operatorname{sat}_{m}^{M}(x)=\left\{\begin{array}{lll}
M & & x \geq M \\
x & \text { if } \quad x \in] m, M[ \\
m & & x \leq m
\end{array}\right.
$$

To simplify the notation, we will write $s a t^{M}(x)$ instead of $s a t_{-M}^{M}(x)$ when $m+M=0$. The results described in this paper could in fact be adapted to a more general class of saturation functions, including those based on the use of tanh. Define also the discontinuous $\operatorname{sign}_{m}^{M}$ function as follows

$$
\operatorname{sign}_{m}^{M}(x)=\left\{\begin{array}{lll}
M & & x \geq 0 \\
0 & \text { if } & x=0 \\
m & & x \leq 0
\end{array}\right.
$$

One remarks that $\operatorname{sign}_{m}^{M}(\cdot)=\lim _{k \rightarrow+\infty} \operatorname{sat}_{m}^{M}(k \cdot)$. Consider the first order integrator

$$
\dot{x}=u \text {, }
$$

with the control variable $u$ such that $m \leq u \leq M$. The TOC associated with this system that takes $x$ to zero in minimal time can be written as

$$
u(x)=\operatorname{sign}_{m}^{M}(-x) .
$$


Due to the discontinuity at the desired set-point, this is not a good feedback law. Indeed, even though it theoretically stabilizes $x=0$ asymptotically (when considering solutions of the controlled system defined in the sense of Filippov, for instance), it is excessively sensitive to measurement noise and chattering, and its discretization systematically renders the origin unstable. A continuous approximation of this optimal control, endowed with better robustness properties around the origin, is given by

$$
u(x)=s a t_{m}^{M}\left(-k_{p} x\right), \quad k_{p}>0,
$$

with the approximation improving uniformly (in terms of reaching a given small neighborhood of $x=0$ from any initial condition) by increasing the value of $k_{p}$. Locally, near the origin, this latter control is equal to the proportional feedback law

$$
u(x)=-k_{p} x .
$$

It thus locally inherits the properties of this linear feedback control, whereas it approximates the TOC when the "error" $x$ is initially large. In practice, the gain $k_{p}$ can be tuned according to Linear Control Theory rules, typically in relation to control sampling, measurement noise, and additive perturbation issues addressed by control performance and robustness analyses.

\section{B. Second-order system}

Consider now the second-order integrator

$$
\ddot{x}=u,
$$

with the same bound constraints as previously, i.e. $m \leq u \leq M$. Set

$$
s_{a}(x, \dot{x})= \begin{cases}x+\frac{\dot{x}|\dot{x}|}{2 a} & x+\frac{\dot{x}|\dot{x}|}{2 a} \neq 0 \\ \dot{x} & \text { if } \quad x+\frac{\dot{x}|\dot{x}|}{2 a}=0, x^{2}+\dot{x}^{2} \neq 0 \\ 0 & x+\frac{\dot{x}|\dot{x}|}{2 a}=0, x^{2}+\dot{x}^{2}=0\end{cases}
$$

The TOC associated with this system that takes $x$ to zero in minimal time can be written as (see, e.g., [2], [4])

$$
u(x, \dot{x})=\operatorname{sign}_{m}^{M}\left(-s_{a}(x, \dot{x})\right), \text { with } a=\left\{\begin{array}{lll}
M & \text { if } & x \geq 0 \\
-m & \text { if } & x<0
\end{array}\right.
$$

a simplification of which is

$$
u(x, \dot{x})=\operatorname{sign}_{m}^{M}\left(-\left(x+\frac{\dot{x}|\dot{x}|}{2 a}\right)\right), \text { with } a= \begin{cases}M & \text { if } \quad x \geq 0 \\ -m & \text { if } x<0\end{cases}
$$

This feedback law is discontinuous at points $(x, \dot{x})$ where $x+\frac{\dot{x}|\dot{x}|}{2 a}=0(a=M,-m)$, and also on the line $x=0$. It is in particular discontinuous at the desired equilibrium $(x, \dot{x})=(0,0)$. In order to ensure continuity at this point one may consider the following approximation:

$$
u(x, \dot{x})=s a t_{m}^{M}\left(-k_{p}\left(x+\frac{\dot{x}|\dot{x}|}{2 a}\right)\right), \text { with } a= \begin{cases}M & \text { if } \quad x \geq 0 \\ -m & \text { if } x<0\end{cases}
$$


where $k_{p}>0$ plays the role of a "proportional gain". Going further in this direction, an approximation which is continuous everywhere is given by

$$
u(x, \dot{x})=s a t_{m}^{M}\left(-k_{p}\left(x+\frac{\dot{x}|\dot{x}|}{2 a(x, \varepsilon)}\right)\right),
$$

with

$$
a(x, \varepsilon)=\frac{M-m}{2}+\frac{M+m}{2} \operatorname{sat}^{1}\left(\frac{x}{\varepsilon}\right),
$$

and $\varepsilon$ a (small) positive number. Note that $a(x, \varepsilon)$ is constant and equal to $M$ in the classical case when $M+m=0$. Now, a shortcoming of the above approximations is that they do not yield a (local) rate of convergence uniformly as fast as exponential, due to the quadratic velocity correction term involved in the time-optimal feedback law. This issue can be taken care of by adding a complementary linear velocity term as follows

$$
u(x, \dot{x})=s a t_{m}^{M}\left(-k_{p}\left(x+\frac{\dot{x}|\dot{x}|}{2 a(x, \varepsilon)}\right)-\operatorname{sat}^{l}\left(k_{v} \dot{x}\right)\right),
$$

with $l>0$ bounding the interval on which this linear term is the most active, and $k_{v}>0$ playing the role of a "derivative gain". Indeed, the linear approximation of the above feedback at the desired equilibrium $(x=0, \dot{x}=0)$ is the classical PD controller

$$
u(x, \dot{x})=-k_{p} x-k_{v} \dot{x},
$$

whose proportional and derivative gains, $k_{p}$ and $k_{v}$, can be determined by applying classical rules of Linear Control Theory. For instance, for the double integrator system $\ddot{x}=u$, the choice $k_{v}=2 \sqrt{k_{p}}$ yields two closed-loop poles equal to $-\sqrt{k_{p}}$ and ensures a criticallydamped response with no overshoot. As for the choice of the parameter $l$, it corresponds to a compromise between (local) robustness (as provided by a linear PD feedback) and performance when starting far away from the desired equilibrium (as provided by the timeoptimal nonlinear control). In [9], Newman studied the particular case where $M+m=0$ and, by considering a sliding-mode formulation, derived a nonlinear PD controller which is essentially the same as (8).

\section{Stability and convergence}

Let us analyze the stability and convergence properties associated with the time suboptimal controller (4) (resp. (8)) applied to the first-order integrator system (3) (resp. second-order integrator system (5)). From the fact that the linear approximations of these controllers coincide with classical P and PD feedbacks one can already deduce that they are local exponential stabilizers. The following lemmas points out that they are in fact global asymptotic stabilizers. In particular, in Lemma 1 we study the case where the function $a(\cdot, \cdot)$ involved in the controller $(8)$ is chosen constant positive. Then, in Lemma 2 the more difficult case with the function $a(\cdot, \cdot)$ defined by $(7)$ is analyzed.

Lemma 1 The nonlinear proportional feedback control (4) globally asymptotically stabilizes $x=0$ for the first-order integrator system (3).

With $a(\cdot, \cdot)$ chosen constant positive, the nonlinear proportional-derivative feedback control (8) globally asymptotically stabilizes $(x, \dot{x})=(0,0)$ for the second-order integrator system (5). 
Proof: The proofs of both lemma's statements is obtained by applying classical Lyapunov function techniques. For the first-order case, consider the function defined by $\mathcal{V}_{1}(x)=$ $0.5 x^{2}$, whose time-derivative along any solution to the closed-loop system is given by $\dot{\mathcal{V}}_{1}=$ $x s a t_{m}^{M}\left(-k_{p} x\right)(\leq 0)$, with the time index omitted for the sake of notation simplification. The resulting boundedness of $\mathcal{V}_{1}(x)$ along any solution to the controlled system yields the stability of the point $x=0$, whereas the convergence of $\dot{\mathcal{V}}_{1}$ to zero yields the convergence of $x$ to zero, i.e. the desired convergence property.

As for the second-order system, let us assume that $a(\cdot, \cdot)$ is positive constant. We note that the control may then be written as $u=s a t_{m}^{M}\left(-\left(k_{p} x+g(\dot{x})\right)\right)$, with

$$
g(s)=\frac{k_{p}|s| s}{2 a}+\operatorname{sat}^{l}\left(k_{v} s\right)
$$

Therefore, $g^{\prime}(s)>\min \left\{k_{v}, \frac{k_{p} l}{k_{v} a}\right\}>0, \forall s$, with $g^{\prime}(s)$ denoting the (right) derivative of $g$ at $s$. Moreover, $s a t_{m}^{M}(s) s>0, \forall s \neq 0$. Consider the positive function

$$
\mathcal{V}_{2}(x, \dot{x})=\frac{k_{p} \dot{x}^{2}}{2}+\int_{0}^{k_{p} x+g(\dot{x})} s a t_{m}^{M}(s) d s,
$$

whose time-derivative along any solution to the closed-loop system is given by

$$
\dot{\mathcal{V}}_{2}=-g^{\prime}(\dot{x})\left(s a t_{m}^{M}\left(-\left(k_{p} x+g(\dot{x})\right)\right)\right)^{2} \leq 0 .
$$

The resulting boundedness of $\mathcal{V}_{2}(x, \dot{x})$ along any solution to the controlled system yields the stability of the point $(x, \dot{x})=(0,0)$. The convergence of $\dot{\mathcal{V}}_{2}$ to zero implies that $s a t_{m}^{M}\left(-\left(k_{p} x+g(\dot{x})\right)\right)$ tends to zero, and thus that $k_{p} x+g(\dot{x})$ tends to zero. Therefore, since $|\dot{x}|$ is bounded, $\frac{d}{d t}\left(x^{2}\right)+\frac{2 g(\dot{x})}{k_{p} \dot{x}} \dot{x}^{2}$ tends to zero. Using the fact that $g(\dot{x}) / \dot{x}>0$, this in turn implies that $x$ and $\dot{x}$ tend to zero.

Remark 1 The function a defined by (7), which is proposed to make the control (8) an approximation of the TOC solution for any initial condition, is positive. It takes its values in the interval comprised between $-m$ and $M$. When $|x|$ is not small, it is (almost) equal to $-m$ if $x$ is negative, and (almost) equal to $M$ if $x$ is positive. This means that in situations where the signs of possible initial values of $x$ are the same (either all positive, or all negative), one can choose $a(x, \varepsilon)$ constant and equal to either $-m$ or $M$, depending on the case, with no performance degradation. A situation of this type occurs with the longitudinal headway car control problem which is addressed in the last part of the paper.

Lemma 2 The nonlinear proportional-derivative feedback control (8), with a $(x, \varepsilon)$ defined by (7) and $0<\varepsilon<\min (-m, M) / k_{p}$, globally asymptotically stabilizes $(x, \dot{x})=(0,0)$ for the second-order integrator system (5).

The proof of this lemma, being long and subtle, is given in Appendix -A.

We have seen so far that the controllers (4) and (8) are exponential stabilizers of the origins of the first-order and second-order integrator systems respectively, that they are continuous approximations of corresponding discontinuous TOCs, and that the approximations are all the better (in terms of functional approximation) than $k_{p}$ is large and $\varepsilon$ is small. As a matter of fact, these feedback controls are well-conditioned alternatives to the original TOCs when it comes to simulate the solutions to the controlled systems by using classical Runge-Kutta numerical integration packages. In practice, of 
course, the use of large control gains poses a number of well-known robustness problems in relation to various implementation issues (modeling errors, control discretization, noise measurement, etc.) so that the tuning of these gains is needed to reach an acceptable performance/robustness compromise. Nevertheless, an important practical shortcoming of these controllers is that they do not preserve the convergence to the desired equilibrium as soon as a non-zero constant - or slowly varying, in practice- additive perturbation acts on the system. Adding an integral action to the control law is the common way to correct this problem. In the next section, we propose a technique to complement the previously derived $\mathrm{P}$ and $\mathrm{PD}$ controllers with such an action, by taking into account the bounds imposed on the control magnitude, with the concerns of limiting wind-up effects and of preserving the global asymptotic stability properties of the original controllers.

\section{INTEGRAL ACTION COMPLEMENTATION}

\section{A. First-order system}

We consider the first-order integrator with a complementary constant (unknown) perturbation input $c$

$$
\dot{x}=u+c .
$$

We further assume that the perturbation magnitude is not too large. More precisely, we assume that $|c| \leq \delta_{c}<\min \{M,-m\}$, with the latter inequality ensuring that the problem of global asymptotic stabilization of $x=0$ has a solution despite the bounds imposed on

the control input. Rather than using a pure integrator of $x$ in the control law, the authors of [12] propose to use a "bounded" integral term $z$ calculated as follows

$$
\dot{z}=k_{z}\left(-z+s a t^{\delta_{z}}(z+x)\right), \quad|z(0)|<\delta_{z},
$$

with $\operatorname{sat}(\cdot)$ denoting the classical saturation function, and $k_{z}$ and $\delta_{z}$ denoting positive numbers. This relation indicates that we have a pure integrator $\dot{z}=k_{z} x$ as long as $|z+x| \leq \delta_{z}$, and also that $|z(t)| \leq \delta_{z}$ and $|\dot{z}(t)| \leq 2 k_{z} \delta_{z}, \forall t \geq 0$. Therefore, by defining $\dot{z}_{\text {max }}$ as the maximal value that $|\dot{z}(t)|$ is allowed to take, one has

$$
\delta_{z}=\frac{\dot{z}_{\max }}{2 k_{z}},
$$

and it is possible to modify the magnitudes of $z$ and $\dot{z}$ at will via the choice of the parameters $\dot{z}_{\max }$ and $k_{z}$. We will see that the first of these parameters characterizes the importance given to the integral action at the control level, whereas $k_{z}$ enters (in a simple way) the calculation of the gains associated with the linear PI controller of which the proposed nonlinear controller is a local approximation at $(x, z)=(0,0)$. Let us proceed with the control design itself. A way to complement the nonlinear proportional feedback (4) with an integral action consists in conceptually replacing the initial state $x$ by the modified state

$$
\bar{x} \equiv x+z,
$$

and determining a control which asymptotically stabilizes the augmented state $(\bar{x}, z)=$ $(0,0)$ when $c \equiv 0$. In this case, and with the above definition of $z$, the augmented control system writes as

$$
\left\{\begin{array}{l}
\dot{\bar{x}}=u+v(\bar{x}, z) \\
\dot{z}=v(\bar{x}, z)
\end{array}\right.
$$

with

$$
v(\bar{x}, z)=k_{z}\left(-z+s a t^{\delta_{z}}(\bar{x})\right) .
$$


The time-suboptimal feedback (4) can be considered to asymptotically stabilize $\bar{x}=0$. Setting

$$
\begin{aligned}
& \dot{z}_{\text {max }}<\min \{M,-m\} \text {, } \\
& \bar{M} \equiv M-\dot{z}_{\max }(>0) \text {, } \\
& \bar{m} \equiv m+\dot{z}_{\max }(<0) \text {, }
\end{aligned}
$$

this yields the feedback controller

$$
\begin{aligned}
u(\bar{x}, z) & =s a t_{\bar{m}}^{\bar{M}}\left(-k_{p} \bar{x}\right)-v(\bar{x}, z) \\
& =s a t_{\bar{m}}^{\bar{M}}\left(-k_{p} \bar{x}\right)-k_{z}\left(-z+\operatorname{sat}^{\delta_{z}}(\bar{x})\right)
\end{aligned}
$$

which, by construction, takes its values in the interval $[m, M]$, and whose linear approximation at $(\bar{x}, z)=(0,0)$ is the linear PI controller $u=-\bar{k}_{p} x-k_{i} \int x$ with $\bar{k}_{p}=k_{p}+k_{z}$ and $k_{i}=k_{p} k_{z}$. The corresponding closed-loop poles are real negative and equal to $-k_{p}$ and $-k_{z}$ respectively.

Lemma 3 The nonlinear PI feedback control (10-13) globally asymptotically stabilizes $(x, z)=\left(0, c / k_{p}\right)$ for the perturbed augmented system (9-10), provided that $0<\dot{z}_{\max }<$ $\min \{-m, M\}-|c|$ and $0<k_{z}<\frac{k_{p} \dot{z}_{\max }}{2 \min \{-m, M\}}$.

Proof: Define $\overline{\bar{x}} \equiv \bar{x}-c / k_{p}$ and $\bar{z} \equiv z-c / k_{p}$. The desired stability property is equivalent to the global asymptotic stabilization of $(\overline{\bar{x}}, \bar{z})=(0,0)$. From the system's equation and control expression, using the fact that

$$
\operatorname{sat}_{m}^{M}(x-\varepsilon)+\varepsilon=s a t_{m+\varepsilon}^{M+\varepsilon}(x),
$$

one easily verifies that along any closed-loop solution

$$
\dot{\overline{\bar{x}}}=s a t_{\bar{m}+c}^{\bar{M}+c}\left(-k_{p} \overline{\bar{x}}\right) .
$$

Using the condition upon $\dot{z}_{\max }$ one deduces that the time-derivative of $\overline{\bar{x}}^{2}$ is negative whenever $\overline{\bar{x}} \neq 0$. This in turn implies that $\overline{\bar{x}}$ tends to zero and that $\overline{\bar{x}}=0$ is globally asymptotically stable. Skipping technical arguments of minor importance, it remains to show that $\bar{z}=0$ is asymptotically stable on the zero dynamics defined by $\overline{\bar{x}}=0$. From (11), using the fact that the condition upon $k_{z}$ implies that $\delta_{z}>|c| / k_{p}$, and thus that sat ${ }^{\delta_{z}}\left(c / k_{p}\right)=c / k_{p}$, the evolution of $\bar{z}$ on this zero dynamics is given by $\dot{\bar{z}}=-k_{z} \bar{z}$. The desired property follows directly.

Choosing $\dot{z}_{\max }$ small does not impede the compensation of perturbations almost as large as the control bounds, and also limits the degradation of the control in terms of time-(sub)optimality. On the other hand, this imposes to use a small gain $k_{z}$ with the risk of much penalizing the ultimate rate of convergence to the equilibrium. With these general rules in mind, the tuning of these parameters will essentially depend on the specific conditions and requirements of the application.

\section{B. Second-order system}

We now consider the second-order integrator with a constant (unknown) perturbation input $c$

$$
\ddot{x}=u+c .
$$

For the same reason as previously we assume that $|c| \leq \delta_{c}<\min \{M,-m\}$. In the case of the first-order system, the relation (10) defines the way the bounded integral term $z$ 
is calculated and ensures that the absolute value of $\dot{z}$ is uniformly bounded by a chosen value. For the second-order system, it is useful -for reasons that will clearly appear further in the paper- to ensure that the second-time derivative of $z$ is uniformly bounded by a chosen value. We propose here to calculate $z$ as follows

$$
\ddot{z}=-k_{v z} \dot{z}+s a t^{\ddot{z}_{\text {max }} / 2}\left(k_{p z}\left(-z+s a t^{\delta_{z}}(z+x)\right)\right)
$$

with $\ddot{z}_{\max }, \delta_{z}, k_{p z}$, and $k_{v z}$ denoting positive numbers, and with initial conditions such that $|z(0)|<\delta_{z}+\ddot{z}_{\max } /\left(2 k_{v z}^{2}\right)$ and $|\dot{z}(0)|<\ddot{z}_{\max } /\left(2 k_{v z}\right)$. One can verify (the proof is left as an exercise to the interested reader) that, whatever the evolution of $x(t)$, the absolute values of $z(t), \dot{z}(t)$, and $\ddot{z}(t)$ are uniformly bounded by $\delta_{z}+\ddot{z}_{\max } /\left(2 k_{v z}^{2}\right), \ddot{z}_{\max } /\left(2 k_{v z}\right)$, and $\ddot{z}_{\max }$ respectively. Away from saturation bounds, the evolution of $z$ is given by $\ddot{z}=-k_{v z} \dot{z}+k_{p z} x$ whose solutions can be approximated by those of the first-order equation $\dot{z}=\left(k_{p z} / k_{v z}\right) x$ when $|\dot{x}|$ is small. This latter equation points out the integral action embedded in (15). Note also that, if $x+z=0$ and $|z|<\ddot{z}_{\max }$, then the evolution of $z$ is given by the autonomous second-order equation $\ddot{z}+k_{v z} \dot{z}+k_{p z} z=0$. Therefore, on the zero dynamics defined by $x+z=0$, the asymptotic exponential rate of convergence of $z$ to zero is proportional to $\omega=\sqrt{k_{p z}}$, whereas $\xi=k_{v z} /\left(2 \sqrt{k_{p z}}\right)$ is the damping factor (typically chosen between 0.7 and 1). Let us proceed with the control design by extending the method used for the first-order case. Using the same definition of $\bar{x}$, i.e. $\bar{x} \equiv x+z$, the augmented control system, in the case where $c \equiv 0$, now writes as

$$
\left\{\begin{array}{l}
\ddot{\bar{x}}=u+v(\bar{x}, z, \dot{z}) \\
\ddot{z}=v(\bar{x}, z, \dot{z})
\end{array}\right.
$$

with

$$
v(\bar{x}, z, \dot{z})=-k_{v z} \dot{z}+s a t^{\ddot{z}_{\max } / 2}\left(k_{p z}\left(-z+s a t^{\delta_{z}}(\bar{x})\right)\right) .
$$

Define

$$
\bar{M} \equiv M-\ddot{z}_{\max }(>0), \bar{m} \equiv m+\ddot{z}_{\max }(<0) .
$$

Using the time sub-optimal PD controller (8) to asymptotically stabilize $(\bar{x}, \dot{\bar{x}})=(0,0)$, with $\bar{M}$ and $\bar{m}$ chosen as control bounds, yields the nonlinear PID feedback control

$$
u(\bar{x}, \dot{\bar{x}}, z, \dot{z})=s a t_{\bar{m}}^{\bar{M}}\left(-k_{p}\left(\bar{x}+\frac{\dot{\bar{x}}|\dot{\bar{x}}|}{2 a(\bar{x}, \varepsilon)}\right)-\operatorname{sat}^{l}\left(k_{v} \dot{\bar{x}}\right)\right)-v(\bar{x}, z, \dot{z})
$$

with $a(\bar{x}, \varepsilon)$ either positive constant or calculated according to (7) with $M$ and $m$ replaced by $\bar{M}$ and $\bar{m}$ respectively. Since $|v(\bar{x}, z, \dot{z})|<\ddot{z}_{\max }$ along any solution to the controlled system, this control takes its values in the interval $[m, M]$. One easily verifies that the linear approximation of the (augmented) closed-loop system at $(\bar{x}, \dot{\bar{x}}, z, \dot{z})=(0,0,0,0)$ is

$$
\left\{\begin{array}{l}
\ddot{\bar{x}}=-k_{p} \bar{x}-k_{v} \dot{\bar{x}} \\
\ddot{z}=-k_{p z} z-k_{v z} \dot{z}+k_{p z} \bar{x}
\end{array}\right.
$$

From these equations one can already deduce that the above controller is a (local) exponential stabilizer of $(\bar{x}, \dot{\bar{x}}, z, \dot{z})=(0,0,0,0)$, and thus also of $(x, \dot{x}, z, \dot{z})=(0,0,0,0)$, when $c \equiv 0$. The following lemmas establish a stronger asymptotic stability property when the perturbation $c$ is not exceedingly large (to the point of rendering the stabilization problem untractable). 
Lemma 4 With $a(\cdot, \cdot)$ chosen positive constant, the nonlinear PID feedback control (1618) globally asymptotically stabilizes $(x, \dot{x}, z, \dot{z})=\left(0,0, c / k_{p}, 0\right)$ for the perturbed augmented system (14-15), provided that $0<\ddot{z}_{\max }<\min \{M,-m\}-|c|$ and $\delta_{z}>|c| / k_{p}$.

Proof: Define $\overline{\bar{x}} \equiv \bar{x}-c / k_{p}$. One easily verifies that along any solution to the controlled system

$$
\ddot{\overline{\bar{x}}}=s a t_{\bar{m}+c}^{\bar{M}+c}\left(-k_{p}\left(\overline{\bar{x}}+\frac{\dot{\overline{\bar{x}}}|\dot{\overline{\bar{x}}}|}{2 a}\right)-s a t^{l}\left(-k_{v} \dot{\bar{x}}\right)\right) .
$$

From this equation and the condition imposed on $\ddot{z}_{\max }$ one deduces, via a minor adaptation of the proof of Lemma 2 , that $(\overline{\bar{x}}, \dot{\bar{x}})=(0,0)$ is globally asymptotically stable. It then suffices to work on the zero dynamics defined by $\overline{\bar{x}}=0$, i.e. $\bar{x}=c / k_{p}$, to prove the global asymptotic stability of $(z, \dot{z})=\left(c / k_{p}, 0\right)$. The technical arguments which justify the previous statement rigorously are classical and omitted for the sake of concision. Define $\bar{z}=z-c / k_{p}$. In view of (15), when $\bar{x}=c / k_{p}$ and $\delta_{z}>|c| / k_{p}$, the evolution of $\bar{z}$ is given by

$$
\ddot{\bar{z}}=-k_{v z} \dot{\bar{z}}+s a t^{\ddot{z}_{\text {max }} / 2}\left(-k_{p z} \bar{z}\right)=-k_{v z} \dot{\bar{z}}-h(\bar{z}) \bar{z}
$$

with $h(\bar{z}) \equiv-(1 / \bar{z}) s a t^{\ddot{z}_{\max } / 2}\left(-k_{p z} \bar{z}\right)(>0, \forall \bar{z})$. Consider the positive function $\mathcal{V}$ defined by $\mathcal{V}(\bar{z}, \dot{\bar{z}})=0.5 \dot{\bar{z}}^{2}+\int_{0}^{\bar{z}} h(s) s d s$. Using the above equation of evolution of $\bar{z}$, the calculation of the time-derivative of this function yields $\dot{\mathcal{V}}=-k_{v z} \dot{\bar{z}}^{2}(<0, \forall \bar{z} \neq 0)$. The stability of $(\bar{z}, \dot{\bar{z}})=(0,0)$, which is equivalent to the stability of $(z, \dot{z})=\left(c / k_{p}, 0\right)$, is a direct consequence of the non-increasing of $\mathcal{V}(\bar{z}, \dot{\bar{z}})$. As for the convergence issue, $\dot{\mathcal{V}}$ tends to zero, and so does $\dot{\bar{z}}$. From there, one shows that $\ddot{\bar{z}}$ is uniformly continuous and thus, by application of Barbalat's Lemma, that $\ddot{\bar{z}}$ tends to zero. In view of the equation of evolution of $\bar{z}$, the convergence of $\dot{\bar{z}}$ and $\ddot{\bar{z}}$ to zero in turn implies that $\bar{z}$ tends to zero.

Lemma 5 With $a(\bar{x}, \varepsilon)$ calculated according to ( 7$)$ with $M$ and $m$ respectively replaced by $\bar{M}$ and $\bar{m}$ (defined by (17)), the nonlinear PID feedback control (16-18) globally asymptotically stabilizes $(x, \dot{x}, z, \dot{z})=\left(0,0, c / k_{p}, 0\right)$ for the perturbed augmented system (14-15), provided that $0<\ddot{z}_{\max }<\min \{M,-m\}-|c|, \delta_{z}>|c| / k_{p}$, and $0<\varepsilon<\frac{\min (-\bar{m}, \bar{M})-|c|}{k_{p}}$.

The proof of this lemma is given in Appendix -B.

A few words concerning the choice of the parameters $\delta_{z}$ and $\ddot{z}_{\max }$ are in order. $\delta_{z}$ should be chosen larger than $c / k_{p}$, as specified in the lemma, but not much larger in order to avoid the possible occurrence of uselessly large values of $z$ favoring large overshoots. As for $\ddot{z}_{\max }$, a compromise has to be found between a small value which minimizes the importance of the integral action, and thus also its negative effects (overshoot and performance degradation in terms of time optimality, in particular), and a larger value which allows for faster desaturation of the integral term $z$.

\section{Application to longitudinal headWAy CAR CONTROL}

Alike other studies on this subject, the control design is here addressed by considering a simple model of the car's dynamics with motorization and braking components schematized to the extreme, the idea being to work out a rough sketch of solutions before going to the stage of adaptation to an actual physical system. The problem statement and modeling equations here considered are basically those of [3], with the noticeable exception of aerodynamic and other drag forces which are not modeled in this reference. We indeed believe that it is important to take these forces into account from the beginning because the intensity of their sum rapidly increases approximately like the square of the 
vehicle' velocity until its reaches it maximal value, corresponding to the vehicle's maximal velocity, when it exactly matches the maximal traction force produced by the vehicle's engine. Let us briefly recall the simplified longitudinal headway control problem that we are addressing:

- the control variable $u$ is the vehicle's acceleration/deceleration capacity. Assuming that the maximal motor-traction force $F_{\text {motor }}$ and braking force $F_{\text {brake }}$ are constant and known, together with the vehicle's mass, one has $u \in[m, M]$ with $m=-\frac{F_{\text {brake }}}{\text { mass }}$ and $M=\frac{F_{\text {motor }}}{\text { mass }}$. - the longitudinal dynamics of the controlled vehicle is given by Newton's law

$$
\ddot{d}=u-k_{d}|v| v
$$

with $d$ denoting the vehicle's abscissa along the road, measured from an arbitrary fixed point, $v=\dot{d}$ the vehicle's velocity, and $k_{d}$ the drag coefficient related to the vehicle's maximal velocity $v_{\max }$ by the relation

$$
v_{\max }^{2}=\frac{M}{k_{d}}
$$

- the leading vehicle's abscissa and velocity are denoted as $d_{r}$ and $v_{r}$ respectively, and the desired inter-distance between the two vehicles, here assumed constant and independent of $v_{r}$ for the sake of simplification, is denoted as $\Delta_{r}$.

Define $x \equiv d-d_{r}+\Delta_{r}$, the control objective is to asymptotically stabilize $x=0$ as efficiently as possible via the calculation of $u$. Combining the above model of the vehicle's dynamics with the definition of $x$ yields the control system

$$
\ddot{x}=u+c\left(\dot{x}, v_{r}, \dot{v}_{r}\right),
$$

with

$$
c\left(\dot{x}, v_{r}\right) \equiv-k_{d}\left|\dot{x}+v_{r}\right|\left(\dot{x}+v_{r}\right)-\dot{v}_{r} .
$$

Although the perturbation $c$ is not constant in this case, it should tend to the constant value $-k_{d}\left|v_{r}\right| v_{r}$ when the leader's velocity $v_{r}$ is constant. If $v_{r}$ varies slowly, it should also vary slowly. This intuitively justifies the idea of applying the nonlinear PID controller (16)-(18) of the previous section.

The simulation results reported below have been performed by assuming that the interdistance $d-d_{r}$ and the difference of velocities $v-v_{r}$ between the two vehicles are (precisely) measured on-line, so that $x$ and $\dot{x}$ are also measured and can be used directly in the control calculation. In practice, the measurement of the inter-distance can be obtained by using various optical devices (cameras, laser range-finders, etc.), but its time-derivative often has to be estimated. Such an estimator is proposed at the end of this section.

For the simulations, we have used the function $a$ given by (7) with $M$ and $m$ replaced by $\bar{M}$ and $\bar{m}$ respectively, and the various parameters involved in the system's dynamics and control calculation have been chosen as follows

- Control bounds: $M=3 \mathrm{~m} / \mathrm{s}^{2}, m=-9 \mathrm{~m} / \mathrm{s}^{2}$

- Drag coefficient and maximal velocity: $k_{d}=1.875 \times 10^{-3} \mathrm{~m}^{-1}, v_{\max }=\sqrt{M / k_{d}}=40 \mathrm{~m} / \mathrm{s}$

- Control gains and other parameters: $k_{p}=k_{p z}=2, k_{v}=k_{v z}=2 \sqrt{k_{p}}, \varepsilon=1, l=20$, $\ddot{z}_{\max }=0.1, \delta_{z}=\left(M-\ddot{z}_{\max }\right) / k_{p}=1.45$. Larger control gains $k_{p}$ and $k_{v}$ would allow for a better approximation of the TOC solution, but would render the control more sensitive to measurement noise and data-acquisition/computation delays.

Moreover, knowing that the integral correction term $z$ is useful only when the error $x$ is not too large, we have modified the calculation of $z$, initially given by (15), as follows

$$
\ddot{z}=-k_{v z} \dot{z}+s a t^{\ddot{z}_{\text {max }} / 2}\left(k_{p z}\left(-z+s a t^{\delta_{z}}\left(z+x \operatorname{bell}_{\nu, s}(x)\right)\right)\right) \text {, }
$$


with

$$
\operatorname{bell}_{\nu, s}(x) \equiv \frac{\tanh ((x+\nu) / s)+\tanh ((-x+\nu) / s)}{2 \tanh (\nu / s)} .
$$

a bell-shaped symmetric function with a peak value equal to one at $x=0$ and which tends to zero when $|x|$ tends to infinity. The parameter $\nu$ characterizes the width of the bell, i.e. the size of the interval in which the contribution of $x$ to the calculation and evolution of $z$ is the most important, whereas $s$ characterizes the steepness of the bell's sides. For the reported simulations we have chosen $\nu=10$ and $s=1$. One easily verifies that this modification only changes the function $h$ in the proof of Lemma 4 without affecting its positivity property on which the result relies.

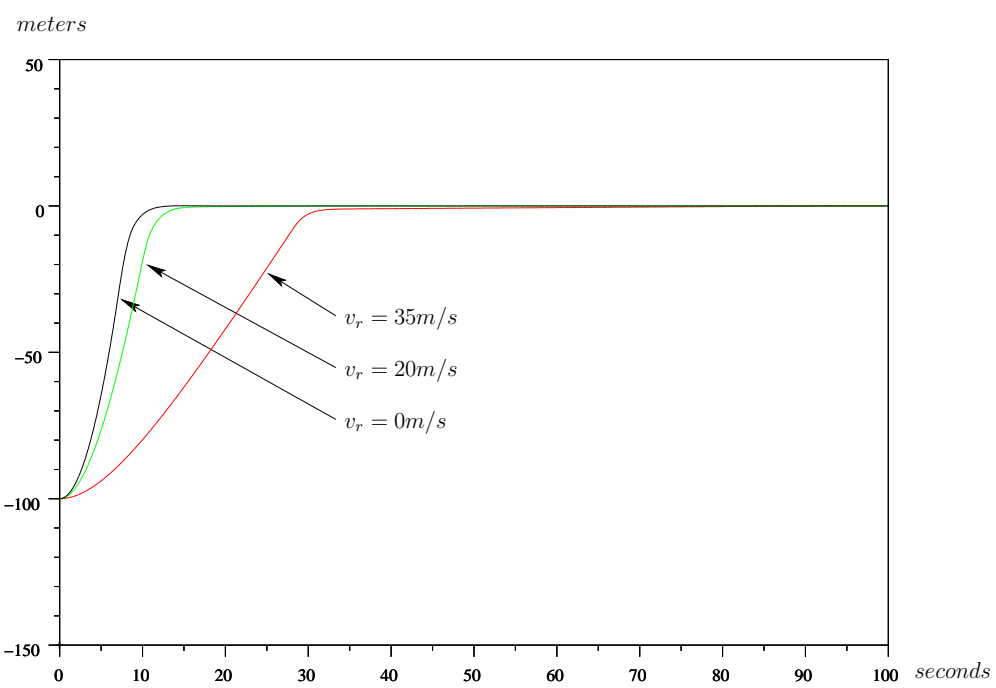

Fig. 1. Inter-distance vs. time

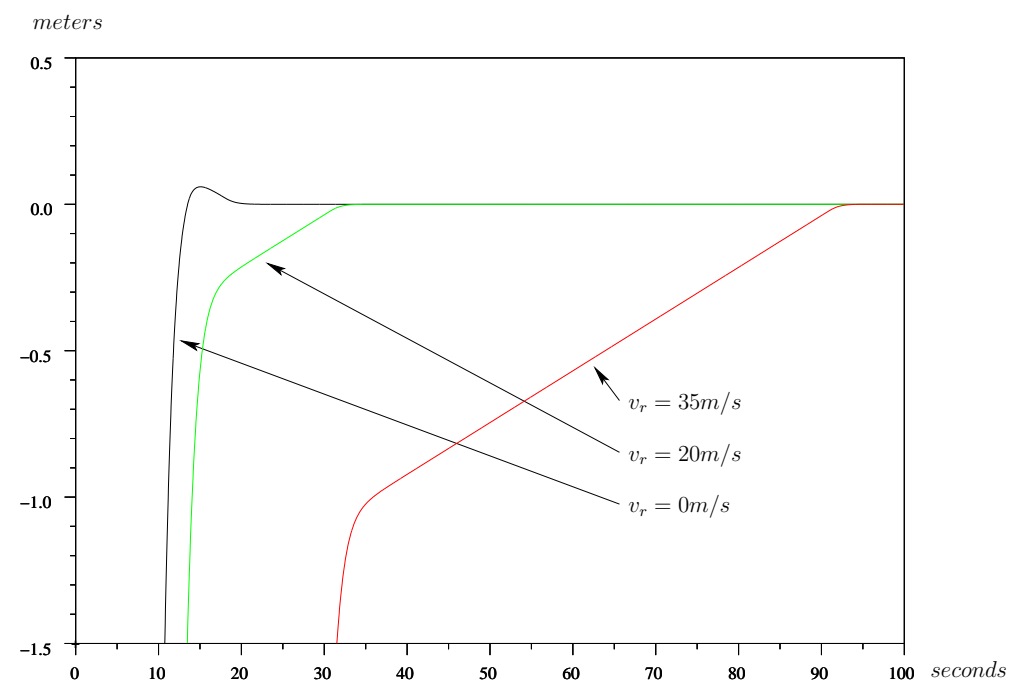

Fig. 2. Inter-distance (zoom) vs. time

The results shown in the figures correspond to three situations involving three distinct leader's velocities $\left(v_{r}=0,20,35 \mathrm{~m} / \mathrm{s}\right)$. The initial inter-distance error is equal to 100 meters in all cases, and the follower's velocity is initially equal to the leader's velocity. The simulation figures show the time-evolution of $i$ ) the inter-distance (Fig. 1 and 2 ), ii) the difference of velocities between the follower and the leader (Fig. 3), iii) the 


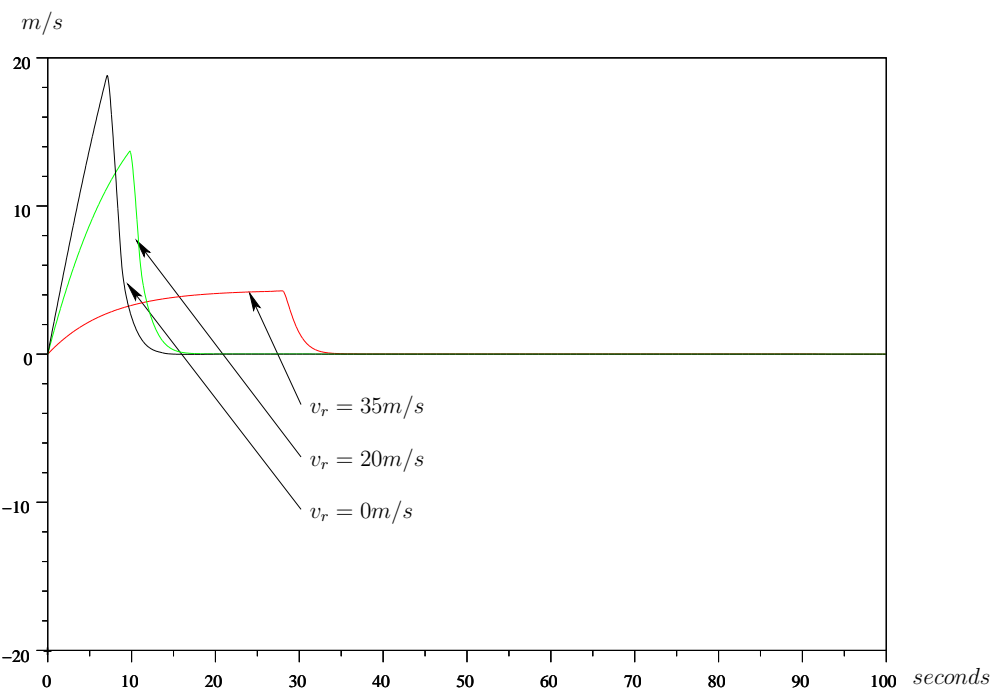

Fig. 3. Velocity difference vs. time

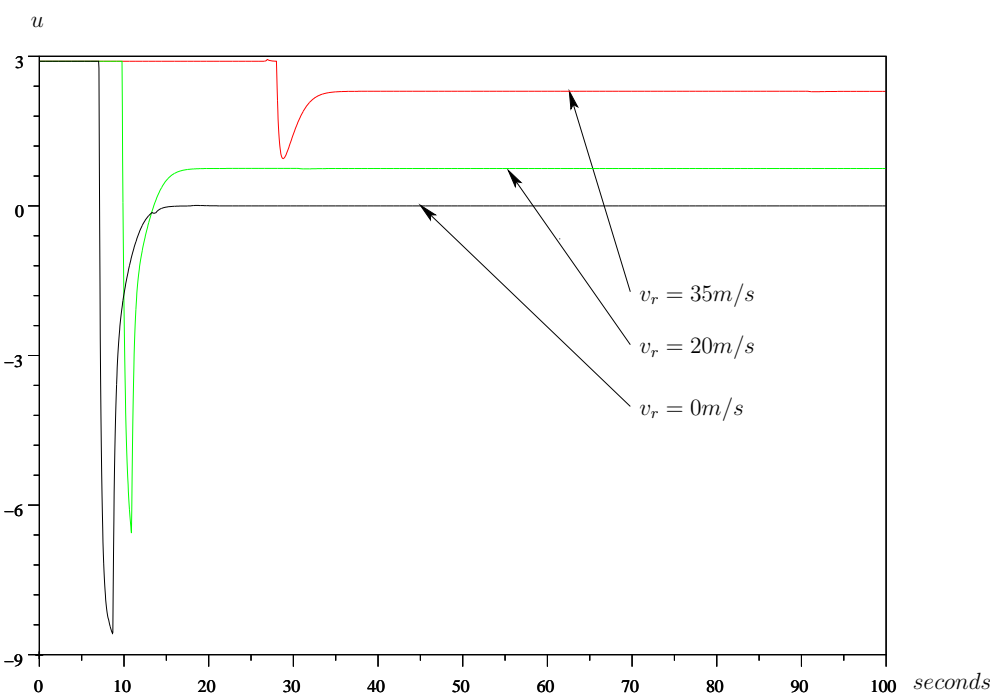

Fig. 4. Control vs. time

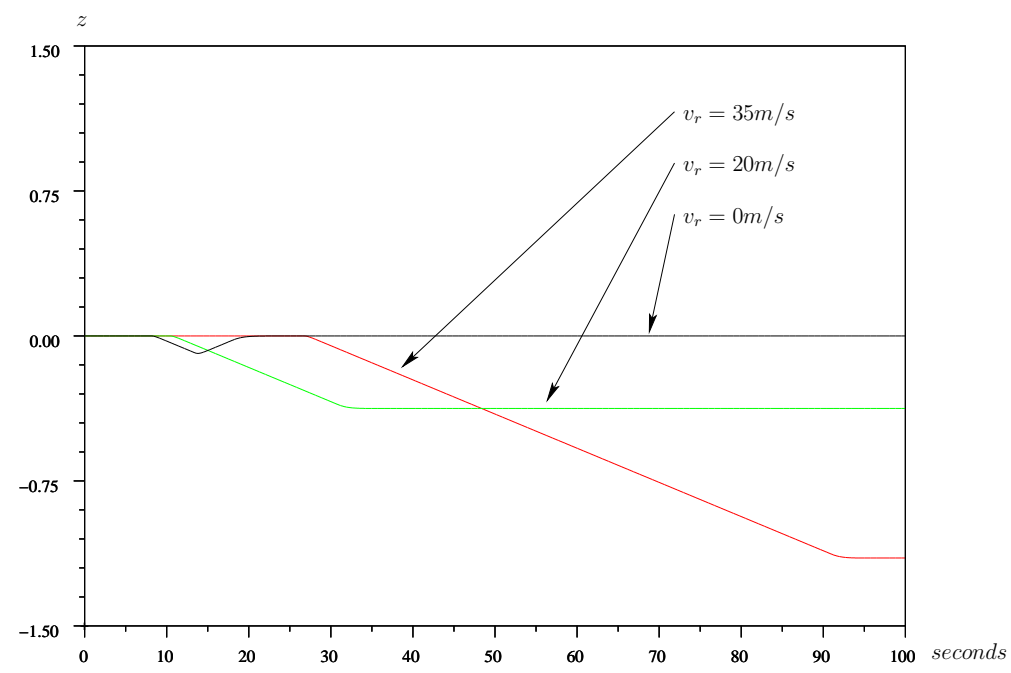

Fig. 5. Integral term $z$ vs. time 
control intensity (Fig. 4), and iv) the correction integral term (Fig. 5). They illustrate important performance differences between the proposed nonlinear PID controller and a (saturated) classical linear PID controller. In particular, the latter would yield important overshoots and several ineffective control-sign changes. One can also observe the consistency and robustness of the system's response for different velocities that involve a large spectrum of drag forces, and the quasi absence of overshoot in all situations, despite the integral correction term which allows for the convergence of tracking error to zero. We also note that for large values of $|x|$ the sign of $x$ is always negative. This is coherent with the context of the application according to which the control is used to "catch up" with the leader, prior to stabilizing the inter-distance distance at zero. Therefore, as pointed out in a previous remark, the control can be implemented with $a$ constant and equal to $-\bar{m}$ without a significant change in performance. However, in this latter case the control would not be optimal for (large) positive initial values of $x$-a virtual possibility, since it means that the leader is initially (far) behind the follower.

For the sake of completeness, let us mention that an on-line estimation $\Delta_{v}$ of $\dot{x}$ based on the measurement of the inter-distance $x$ is obtained by considering the model $\ddot{x}=u+c$ -with $c$ denoting a constant but unknown perturbation- for which a state estimator is given by

$$
\left\{\begin{array}{l}
\dot{\Delta}_{v}=k_{1}(x-\hat{x})-k_{2} w+u \\
\dot{w}=-k_{3}(x-\hat{x}) \\
\dot{\hat{x}}=\Delta_{v}+k_{4}(x-\hat{x})
\end{array}\right.
$$

with $k_{i}(i=1, \ldots, 4)$ denoting positive numbers. One easily verifies that the characteristic polynomial associated with the estimation-error model is $P(\lambda)=\lambda^{3}+k_{4} \lambda^{2}+k_{1} \lambda+k_{2} k_{3}$. Therefore, the transient performance of the estimator can be tuned via the choice of these coefficients, in relation to the poles of the linear approximation of the controlled system near the desired equilibrium. For instance, by setting $k_{4}=3 \omega, k_{1}=3 \omega^{2}$, and $k_{2} k_{3}=\omega^{3}$, with $\omega>0$, the three roots of the characteristic polynomial are real and equal to $-\omega$. Then, the larger $\omega$, the faster the estimation convergence rate, and the smaller the estimation error when the inter-distance velocity is not constant. However, in practice, the size of $\omega$ is limited by common high-gain sensitivity to noise/delay effects. Simulations that we have performed with $\omega=2$ and $k_{3}=1$, and by using $\Delta_{v}$ instead of $\dot{x}$ in the control expression, did not show a significant difference with the case where $\dot{x}$ is directly measured.

\section{REFERENCES}

[1] K.J. Aström and T. Hägglund. PID Controllers: Theory, Design and Tuning. Research Triangle Park, NC: Instrument Society of America, 1995.

[2] M. Athans and P. Falb. Optimal Control. McGraw-Hill Book Company, Prentice Hall, 1966.

[3] C. Hatipoğlu, Ü. Özgüner, and M. Sommerville. Adaptive proximate time-optimal servomechanisms: continuous time case. In IEEE Int. Conf. on Control Applications, Dearborn, pages 721-726, 1996.

[4] D.E. Kirk. Optimal Control Theory. Prentice Hall, 1970.

[5] H.N. Koivo and J.T. Tanttu. Tuning of pid controllers: Survey of siso and mimo techniques. In Proc. IFAC Intelligent Tuning and Adaptive Control Symposium, pages 75-80, 1991.

[6] M. V. Kothare, P. J. Campo, M. Morari, and C. N. Nett. A unified framework for the study of anti-windup designs. Automatica, 30(12):1869-1883, 1994.

[7] L. Marconi and A. Isidori. Robust global stabilization of a class of uncertain feedforward nonlinear systems. Systems and Control Letters, 41:281-290, 2000.

[8] J.-J. Martinez and C. Canudas de Wit. A safe longitudinal control for adaptive cruise control and stop-and-go scenarios. IEEE Trans. on Control Systems Technology, 15(2):246-258, 2007.

[9] W.S. Newman. Robust near time-optimal control. IEEE Trans. on Automatic Control, 35(7):841-844, 1990.

[10] A. O'Dwyer. Handbook of PI and PID controller tuning rules, 3rd edition. Imperial College Press, World Scientific Publishing Co. Pte. Ltd., 2009. 
[11] L.Y. Pao and G.F. Franklin. Proximate time-optimal control of third-order servomechanisms. IEEE Trans. on Automatic Control, 38(4):560-580, 1993.

[12] S. Seshagiri and H. Khalil. Robust output feed back regulation of minimum-phase nonlinear systems using conditional integrators. Automatica, 41:43-54, 2005.

[13] A.R. Teel. Global stabilization and restricted tracking for multiple integrators with bounded controls. Systems and Control Letters, 18:165-171, 1992.

[14] Y. Wang and R.-N. Ma. Global stabilization of feedforward nonlinear system based on nested saturated control. Acta Automatica Sinica, 36(4):528-533, 2010.

[15] M.L. Workman, R.L. Kosut, and G.F. Franklin. Adaptive proximate time-optimal servomechanisms: continuous time case. In American Control Conference (ACC), 6th., Minneapolis, pages 589-594, 1987.

[16] Y. Zhang, B. Kosmatopoulos, P.A. Ioannou, and C.C. Chien. Autonomous intelligent cruise control using front and back information for tight vehicle following maneuvers. IEEE Trans. on Vehicular Technology, 48(1):319-328, 1999.

\section{APPENDIX}

A. Proof of Lemma 2

The closed-loop system writes

$$
\ddot{x}=s a t_{m}^{M}\left(-k_{p} x-g(\dot{x}, x)\right),
$$

with $g(\dot{x}, x)=\frac{k_{p} \dot{x}|\dot{x}|}{2 a(x, \varepsilon)}+s a t^{l}\left(k_{v} \dot{x}\right)$. First, one easily verifies the following facts:

F.1) $\operatorname{sign}(g(\dot{x}, x))=\operatorname{sign}(\dot{x})$,

F.2) $\Delta \equiv \min (-m, M) \leq a(x, \varepsilon) \leq \max (-m, M)$,

F.3) $|x|>\varepsilon \Rightarrow \frac{\partial a}{\partial x}(x, \varepsilon)=\frac{\partial g}{\partial x}(\dot{x}, x)=0$.

Then, let us prove the following properties

Property 1 The solution $(x(t), \dot{x}(t))$ to the closed-loop system (23) are uniformly bounded w.r.t. initial conditions and uniformly continuous.

Property 2 If $\dot{x}$ does not converge to zero, then $\forall t_{0}, \forall e>0, \exists t>t_{0}$ such that $|x(t)|>$ $\Delta / k_{p}-e$.

Proof of Properties 1 and 2: Consider the following positive function

$$
\mathcal{U}(x, \dot{x})=-\frac{1}{k_{p}} \int_{0}^{k_{p} x} s a t_{m}^{M}(-s) d s+\frac{\dot{x}^{2}}{2}
$$

whose time-derivative along any solution to (23) is given by

$$
\dot{\mathcal{U}}=-\dot{x} s a t_{m}^{M}\left(-k_{p} x\right)+\dot{x} s a t_{m}^{M}\left(-k_{p} x-g(\dot{x}, x)\right) .
$$

Now, we will prove that $\dot{\mathcal{U}} \leq 0, \forall(x, \dot{x})$, by considering the following cases and using Fact F.1:

i) Case $\dot{x} x=0$ : One has $\dot{\mathcal{U}}=0$ if $\dot{x}=0$, and $\dot{\mathcal{U}}=\dot{x} s a t_{m}^{M}(-g(\dot{x}, x)) \leq 0$ if $x=0$.

ii) Case $\dot{x} x>0$ : One verifies that $\left|k_{p} x+g(\dot{x}, x)\right| \geq\left|k_{p} x\right|$ and $\operatorname{sign}\left(k_{p} x+g(\dot{x}, x)\right)=$ $\operatorname{sign}(\dot{x})$. As a consequence, one has $\dot{x} s a t_{m}^{M}\left(-k_{p} x-g(\dot{x}, x)\right) \leq \dot{x} s a t_{m}^{M}\left(-k_{p} x\right)$ or equivalently $\dot{\mathcal{U}} \leq 0$.

iii) Case $\dot{x} x<0$ and $\operatorname{sign}\left(k_{p} x+g(\dot{x}, x)\right) \neq \operatorname{sign}(x)$ : It is straightforward to verify that $-\dot{x} s a t_{m}^{M}\left(-k_{p} x\right)<0$ and $\dot{x} s a t_{m}^{M}\left(-k_{p} x-g(\dot{x}, x)\right) \leq 0$, and subsequently $\dot{\mathcal{U}}<0$.

iv) Case $\dot{x} x<0$ and $\operatorname{sign}\left(k_{p} x+g(\dot{x}, x)\right)=\operatorname{sign}(x)$ : One verifies that $-\dot{x} s a t_{m}^{M}\left(-k_{p} x\right)<0$ and $\dot{x} s a t_{m}^{M}\left(-k_{p} x-g(\dot{x}, x)\right)>0$. Besides, one also verifies that $\left|k_{p} x+g(\dot{x}, x)\right|<\left|k_{p} x\right|$ which implies that $\left|\dot{x} s a t_{m}^{M}\left(-k_{p} x-g(\dot{x}, x)\right)\right|<\left|\dot{x} s a t_{m}^{M}\left(-k_{p} x\right)\right|$. Subsequently, one deduces $\dot{\mathcal{U}}<0$. 
The fact that $\dot{\mathcal{U}} \leq 0$ implies that $\mathcal{U}$ is non-increasing. Thus, $x$ and $\dot{x}$ are uniformly bounded w.r.t. initial conditions. The boundedness of $\dot{x}$ implies that $x$ is uniformly continuous. It also results from the boundedness of $x$ and $\dot{x}$ that $\ddot{x}$ is itself bounded, and thus that $\dot{x}$ is uniformly continuous (end of proof of Property 1 ).

Now, let us prove Property 2 by contradiction. Assume that there exist $t_{0}>0$ and $e>0$ such that $|x(t)| \leq \Delta / k_{p}-e, \forall t \geq t_{0}$. Then, sat $t_{m}^{M}\left(-k_{p} x(t)\right)=-k_{p} x(t)$ and $s a t_{m}^{M}\left(-k_{p} x(t)-\right.$ $g(\dot{x}(t), x(t)))=-k_{p} x(t)-\lambda(t) g(\dot{x}(t), x(t))$, with $0<\left(k_{p} e\right) / \sup _{t \geq 0} g(\dot{x}(t), x(t)) \leq \lambda(t) \leq$ $1, \forall t \geq t_{0}$. Therefore,

$$
\dot{\mathcal{U}}(t) \leq-\lambda(t) \dot{x}(t) g(\dot{x}(t), x(t)) \leq-\lambda(t) \frac{k_{p}}{2 \max (-m, M)}|\dot{x}(t)|^{3} \leq 0, \forall t \geq t_{0} .
$$

This inequality, combined with the positivity of $\mathcal{U}(t)$, in turn implies that $\dot{\mathcal{U}}(t)$ and thus $\dot{x}(t)$ converges to zero. Property 2 is a direct consequence of the resulting contradiction (end of proof of Property 2).

Property $3 \forall t_{0}, \exists t>t_{0}$ such that $|x(t)| \leq \varepsilon$.

Proof of Property 3: Consider the positive function

$$
\mathcal{V}(x, \dot{x})=\frac{1}{k_{p}} \int_{0}^{k_{p} x+g(\dot{x})} s a t_{m}^{M}(s) d s+\frac{\dot{x}^{2}}{2},
$$

and consider a solution $(x(t), \dot{x}(t))$ to $(23)$. Assume that $|x(t)|>\varepsilon$ for all $t$ larger than some time instant $t_{0}$. Then, in view of Fact F.3 one has $\forall t>t_{0}$

$$
\dot{\mathcal{V}}(t)=-\frac{\partial g}{\partial \dot{x}}(\dot{x}(t), x(t))\left(s a t_{m}^{M}\left(-k_{p} x(t)-g(\dot{x}(t), x(t))\right)\right)^{2},
$$

with $\frac{\partial g}{\partial \dot{x}}(\dot{x}(t), x(t))>\min \left\{k_{v}, \frac{k_{p} l}{k_{v} \max (-m, M)}\right\}>0$. Therefore, $\dot{\mathcal{V}}(t)$ is non-positive and converges to zero. This in turn implies that $k_{p} x(t)+g(\dot{x}(t), x(t))$ converges to zero. Then, similarly to the proof of Lemma 1 one deduces that $x(t)$ and $\dot{x}(t)$ also converge to zero. Property 3 is a direct consequence of the resulting contradiction (end of proof of Property 3).

Now, let us prove Lemma 2. The stability of $(x, \dot{x})=(0,0)$ results from the already proven boundedness of $\mathcal{U}$ defined by $(24)$ w.r.t. initial conditions. It thus remains to prove that any solution $(x(t), \dot{x}(t))$ to $(23)$ converges to $(0,0)$. Pick any positive number $e$ smaller than $\left(\Delta / k_{p}-\varepsilon\right) / 2$, i.e. $0<e<\left(\Delta / k_{p}-\varepsilon\right) / 2$. Let us assume that $\dot{x}$ does not converges to zero. Then, Properties 2 and 3 ensure the existence of a sequence of time interval $S_{i} \equiv\left[t_{i, 1}, t_{i, 2}\right]$, with $i \in \mathbb{N}$, such that

- $t_{i, 1}<t_{i, 2}<t_{i+1,1}$,

- $x\left(t_{i, 1}\right)= \pm \varepsilon$,

- $x\left(t_{i, 2}\right)= \pm\left(\Delta / k_{p}-e\right)$,

- $\forall t \in\left[t_{i, 1}, t_{i, 2}\right]:|x(t)| \leq \Delta / k_{p}-e$.

Note that the existence of this sequence and the fact that $\dot{x}$ is bounded also implies that $t_{i, 1}$ tends to infinity with $i$. It is also important to remark that if $\dot{x}$ does not converges to zero, then any solution to (23) enters regularly (infinitely of time) the domain $|x| \leq \Delta / k_{p}-e$ and remains in this domain for a strictly positive amount of time.

Property 4 If $\dot{x}$ does not converge to zero, then $\exists \varepsilon_{v}>0$, such that $\forall i \in \mathbb{N}, \forall t \in\left[t_{i, 1}, t_{i, 2}\right]$ one has $|\dot{x}(t)| \geq \varepsilon_{v}$. 
Proof of Property 4: Assume that there exists $i \in \mathbb{N}$ and $t_{i, 3} \in\left[t_{i, 1}, t_{i, 2}\right]$ such that $\left|\dot{x}\left(t_{i, 3}\right)\right|<$ $\varepsilon_{v}:=\sqrt{e\left(2 \Delta-k_{p} e\right)}$, then

$$
\begin{aligned}
\mathcal{U}\left(t_{i, 3}\right) & =-\frac{1}{k_{p}} \int_{0}^{k_{p} x\left(t_{i, 3}\right)} s a t_{m}^{M}(-s) d s+\frac{\dot{x}\left(t_{i, 3}\right)^{2}}{2}=\frac{1}{k_{p}} \int_{0}^{k_{p} x\left(t_{i, 3}\right)} s d s+\frac{\dot{x}\left(t_{i, 3}\right)^{2}}{2} \\
& <\frac{k_{p}}{2}\left(\frac{\Delta}{k_{p}}-e\right)^{2}+\frac{e\left(2 \Delta-k_{p} e\right)}{2}=\frac{\Delta^{2}}{2 k_{p}}=-\frac{1}{k_{p}} \int_{0}^{\Delta} s a t_{m}^{M}(-s) d s .
\end{aligned}
$$

This strict inequality implies the existence of some constant $\bar{e}>0$ such that

$$
\mathcal{U}\left(t_{i, 3}\right) \leq-\frac{1}{k_{p}} \int_{0}^{\Delta-k_{p} \bar{e}} s a t_{m}^{M}(-s) d s .
$$

Since $\mathcal{U}(t)$ is non-increasing, $\forall t \geq t_{i, 3}$ one has

$$
-\frac{1}{k_{p}} \int_{0}^{k_{p} x(t)} s a t_{m}^{M}(-s) d s \leq \mathcal{U}(t) \leq \mathcal{U}\left(t_{i, 3}\right) \leq-\frac{1}{k_{p}} \int_{0}^{\Delta-k_{p} \bar{e}} s a t_{m}^{M}(-s) d s .
$$

Therefore, $|x(t)|<\Delta / k_{p}-\bar{e}, \forall t \geq t_{i, 3}$. The resulting contradiction with Property 2 yields Property 4 (end of proof of Property 4 ).

Now, using Property 4 , one has $\forall t \in\left[t_{i, 1}, t_{i, 2}\right]$

$$
\begin{aligned}
\dot{\mathcal{U}}(t) & =-\dot{x}(t) s a t_{m}^{M}\left(-k_{p} x(t)\right)+\dot{x}(t) s a t_{m}^{M}\left(-k_{p} x(t)-g(\dot{x}(t), x(t))\right) \\
& =k_{p} \dot{x}(t) x(t)+\dot{x}(t)\left(-k_{p} x(t)+s a t_{m+k_{p} x(t)}^{M+k_{p} x(t)}(-g(\dot{x}(t), x(t)))\right) \\
& =\dot{x}(t) s a t_{m+k_{p} x(t)}^{M+k_{p} x(t)}(-g(\dot{x}(t), x(t))) .
\end{aligned}
$$

Then, using the fact that

$$
m+k_{p} x(t) \leq-\Delta+k_{p} x(t)<-k_{p} e<k_{p} e<\Delta+k_{p} x(t)<M+k_{p} x(t),
$$

one ensures that, $\forall t \in\left[t_{i, 1}, t_{i, 2}\right]$,

$$
\dot{\mathcal{U}}(t) \leq-\dot{x}(t) \operatorname{sat}^{k_{p} e}(g(\dot{x}(t), x(t))) \leq \gamma \varepsilon_{v}^{2},
$$

for some positive constant $\gamma$. Moreover, each time interval $\left(t_{i, 2}-t_{i, 1}\right)$ is larger than some positive constant $\delta$, due to the boundedness of $\dot{x}$. Therefore, using (26) and the fact that $\mathcal{U}$ in non-increasing one deduces that

$$
\mathcal{U}(+\infty) \leq \mathcal{U}(0)-\sum_{i=0}^{+\infty} \delta \gamma \varepsilon_{v}^{2}=-\infty
$$

which contradicts with the fact that $\mathcal{U}$ is a positive function.

One deduces from this contradiction that the initial assumption according to which $\dot{x}$ does not converge to zero is not true. Therefore, $\dot{x}$ tends to zero. By Barbalat's lemma, $\ddot{x}$ also tends to zero, since $\dot{x}$ is uniformly continuous. Finally, in view of (23) the convergence of $\dot{x}$ and $\ddot{x}$ to zero is possible only if $x$ tends to zero. 


\section{B. Proof of Lemma 5}

Define $\overline{\bar{x}} \equiv \bar{x}-c / k_{p}$. One verifies that along any solution to the controlled system

$$
\ddot{\overline{\bar{x}}}=s a t_{\bar{m}+c}^{\bar{M}+c}\left(-k_{p} \overline{\bar{x}}-g(\dot{\bar{x}}, \overline{\bar{x}})\right)
$$

with

$$
g(\dot{\bar{x}}, \overline{\bar{x}})=\frac{k_{p} \dot{\overline{\bar{x}}}|\dot{\overline{\bar{x}}}|}{2 a(\overline{\bar{x}}, \varepsilon)}+s a t^{l}\left(-k_{v} \dot{\bar{x}}\right)
$$

and

$$
a(\overline{\bar{x}}, \varepsilon)=\frac{\bar{M}-\bar{m}}{2}-\frac{\bar{M}+\bar{m}}{2} s a t^{1}\left(\frac{\overline{\bar{x}}+c / k_{p}}{\varepsilon}\right) .
$$

From here the proof proceeds similarly to the proof of Lemma 2 to deduce that $\overline{\bar{x}}$ and $\dot{\bar{x}}$ tend to zero. For instance, Facts F.1-F.3 (in the proof of Lemma 2) can be modified as F.1) $\operatorname{sign}(g(\dot{\overline{\bar{x}}}, \overline{\bar{x}}))=\operatorname{sign}(\dot{\overline{\bar{x}}})$,

F.2) $\Delta \equiv \min (-\bar{m}, \bar{M}) \leq a(\overline{\bar{x}}, \varepsilon) \leq \max (-\bar{m}, \bar{M})$,

F.3) If $\overline{\bar{x}}>\varepsilon-c / k_{p}$ or $\overline{\bar{x}}<-\varepsilon-c / k_{p}$, then $\left.\frac{\partial a}{\partial \overline{\bar{x}}}(\overline{\bar{x}}, \varepsilon)=\frac{\partial g}{\partial \overline{\bar{x}}}(\dot{\overline{\bar{x}}}, \overline{\bar{x}})\right)=0$.

Properties 1 and 2 still hold with $x$ and $\dot{x}$ replaced by $\overline{\bar{x}}$ and $\dot{\overline{\bar{x}}}$ respectively, and system (23) replaced by (27). Note that the positive function $\mathcal{U}$ defined by (24) is used for the proof of these properties, where $x$ and $\dot{x}$ are replaced by $\overline{\bar{x}}$ and $\dot{\overline{\bar{x}}}$ respectively, and $m$ and $M$ are replaced by $\bar{m}$ and $\bar{M}$ respectively. Besides, as a consequence of Fact F.3, Property 3 can be restated as:

$$
(P 3): \quad \forall t_{0}, \exists t>t_{0} \text { s.t. }-\varepsilon-c / k_{p} \leq \overline{\bar{x}}(t) \leq \varepsilon-c / k_{p} .
$$

Then, the proof of convergence of $(\overline{\bar{x}}, \dot{\bar{x}})$ to zero follows the same line as the proof of Lemma 2. Pick any positive number $e$ smaller than $\frac{1}{2}\left(\frac{\Delta-|c|}{k_{p}}-\varepsilon\right)$. Note that conditions on $\ddot{z}_{\text {max }}$ and $\varepsilon$ ensure the existence of such a number $e$. Let us assume that $\dot{\overline{\bar{x}}}$ does not converges to zero. Then, Properties 2 and 3 ensure the existence of a sequence of time interval $S_{i} \equiv\left[t_{i, 1}, t_{i, 2}\right]$, with $i \in \mathbb{N}$, such that

- $t_{i, 1}<t_{i, 2}<t_{i+1,1}$,

- $x\left(t_{i, 1}\right)$ equal to either $\varepsilon-c / k_{p}$ or $-\varepsilon-c / k_{p}$,

- $x\left(t_{i, 2}\right)= \pm\left(\Delta / k_{p}-e\right)$,

- $\forall t \in\left[t_{i, 1}, t_{i, 2}\right]:|x(t)| \leq \Delta / k_{p}-e$.

Note that the condition on $e$ ensures that

$$
-\left(\frac{\Delta}{k_{p}}-e\right)<-\varepsilon-\frac{c}{k_{p}}<\varepsilon-\frac{c}{k_{p}}<\frac{\Delta}{k_{p}}-e,
$$

which is important to ensure the validity of the fourth property of the sequence of time interval $S_{i}$. This in turn ensures that Property 4 , with $\dot{x}$ replaced by $\dot{\bar{x}}$, is still valid. From here, one proceeds exactly like the proof of Lemma 2 to find a contradiction on the assumption that $\dot{\bar{x}}$ does not converges to zero, and deduce, subsequently, that $(\overline{\bar{x}}, \dot{\overline{\bar{x}}})=(0,0)$ is globally asymptotically stable. From here, the proof of global stability of $(x, \dot{x}, z, \dot{z})=(0,0,0,0)$ follows the same line as the proof of Lemma 4 . 